\title{
Analysis of Comparative Genetic Diversity in Two Cultivated Species of Jute
}

\author{
Anita Roy ${ }^{1}$, Sourav Hazari ${ }^{2 *}$ and Sudip Bhattacharya ${ }^{2}$ \\ ${ }^{1}$ Department of Genetics and Plant Breeding, AINPJ \& AF, Kalyani centre, \\ ${ }^{2}$ Department of Genetics and Plant Breeding, Bidhan Chandra Krishi Viswavidyalaya, \\ W. B., India \\ *Corresponding author
}

\section{A B S T R A C T}

\section{Keywords}

Selection index, diversity, cluster analysis, marketable traits

Article Info

Accepted:

15 December 2019

Available Online:

20 January 2020
Cultivated species of jute (Corchorus olitorius L. and Corchorus capsularis L.) are distinct in their growth and characteristics related to dry matter and fibre yield. The present study was planned to evaluate 77 varieties of jute including two checks, each of $C$. capsularis and $C$. olitorius. Characterizations of genotypes of both the species were also carried out on the basis of five marketable traits with their correlation and cluster analysis of them to study the diversity pattern, based on these characters. However they showed considerable variation which indicates that overall genetic variability in the population and there is enough scope of selection. The most interesting parameter being the dry fibre weight and fibre\%, was found to be more in C.olitorius lines (mean fibre weight was $11.71 \mathrm{~g}$ and mean fibre\% was $6.82 \%$ ) and OIJ-07 recorded the highest fibre\% of 8.28 and also the highest fibre wt of $15.26 \mathrm{~g}$. In case of C.capsularis the mean fibre \% was less (mean 5.83\%). When the correlation was studied about the fibre wt with plant height, basal diameter, green wt and fibre \%, it represented a strong positive correlation with the green weight as expected and in both the cases it was around 0.8-0.9 depicting that the green weight can be taken as indirect index for selection in the advance materials.

\section{Introduction}

Jute is a dicotyledonous fiber-yielding crop, belonging to the genus Corchorus of the family Tiliaceae. The genus comprises 50 to 60 species, including the two fiber-yielding cultivated species, $C$. olitorius (tossa jute) and C. capsularis (white jute). Cultivated species of jute, (Corchorus olitorius L. and Corchorus capsularis L.) are distinct in their growth and characteristics related to dry matter and fibre yield. Genetic evaluation and characterization in both the cultivated species of jute have been reported by several workers but they studied the two area separately (Kumar et al., 2005 and Nayak and Baisakh, 2009) and comparative analysis was not done. Success of plant breeding depends upon the nature and 
magnitude of variability present in the germplasm. Qualitative characters being more stable over generations and environments are reliable for characterization of germplasm. Since jute has been domesticated only around 200 years ago and many mutants have not yet been accumulated in jute population due to lack of human selection pressure for longer time (Mukherjee and Kumar, 2002), qualitative morphological characters of jute are mostly monomorphic and few are dimorphic and polymorphic. In India, while certain diagnostic features for released or notified jute varieties are known and used in seed certification (Kumar et al., 2005) the descriptors by and large are incomplete. The jute varieties have not so far been extensively described for various heritable morphological traits to enable the selection of lines and advance materials. Hence, the present study was planned to evaluate 77 varieties of jute including two checks, each of $C$. capsularis and $C$. olitorius. Characterizations of genotypes of both the species were carried out on the basis of five important morphoagronomical traits with their correlation and cluster analysis. However this also depicts that overall genetic variability is low as earlier from the studies of Banerjee et al., 2012 and there is need for creation of variability through interspecific crosses or mutation.

However Corchorus species are characterized by a high degree of inter-specific variability, but a low level of intra-specific variability. Due to modern method of cultivation, the genetic base of the cultivated varieties has become very narrow, where $C$. olitorius cultivars are dominating in cultivation as compared to $C$. capsularis varieties. Most of the adapted areas of the jute growing region are represented by only a few olitorius varieties, particularly 'JRO 524'. Narrow genetic base in $C$. capsularis is a matter of great concern in varietal development, Kar et al., 2009.

\section{Materials and Methods}

The experimental materials comprising of 75 germplasms \& 2 released verities each of two species (C. olitorius \& C.capsularis) which were collected from AINPJ\&AF. The released varieties of tossa jute and white jute are JRO524 \& JRO-204 and JRC-517 \& JRC-698 respectively. The experimental materials were grown in a randomized block design with three replications at the Mondouri Teaching Farm of Bidhan Chandra Krishi Viswavidyalaya (West Bengal) during 20152016 with plot size $6.0 \times 1.60 \mathrm{~m}^{2}$ with 4 lines in each plot. Row to row and plant to plant distances were $25 \mathrm{~cm}$ and $10 \mathrm{~cm}$, respectively. Standard package of practices were followed to raise the crop. Crop was harvested at 120 days after sowing. Data were recorded for five quantitative characters viz. plant height $(\mathrm{cm})$, basal diameter $(\mathrm{cm})$, green weight $(\mathrm{gm})$, fibre weight $(\mathrm{gm})$ and fibre percentage[(Fibre weight / Green weight $\times 100$ ]. Plant height was recorded as height of the main stem measured from ground level to the point of forking at pre bud stage (before development of first flower). Observations were recorded on 10 randomly selected plants in each of three replications at specified stages of crop growth period when the characters under study had full expression. Genetic analysis was carried out using Excel stat and DARwin software programme.

\section{Results and Discussion}

All the genotypes varied significantly for all the traits under observation in both the species. From the mean performance (Table 4 $\& 5)$ it was observed that highest plant height $(327.33 \mathrm{~cm})$ was observed in case of $C$. olitorius (OIJ 29) followed by OIJ 24 (327.17 $\mathrm{cm})$. By observing overall mean performances (Table 1), plant height of $C$. capsularis genotypes are generally shorter than that of $C$. olitorius as was observed in the mean which 
was $255.6 \mathrm{~cm}$ for white jute and $294.66 \mathrm{~cm}$ in case of tossa jute. Same result observed by Hossain and Sasmal 2006. Highest plant height $(317.2 \mathrm{~cm})$ was observed in case of white jute is (CIJ 01) followed by CIN 22 $(310.7 \mathrm{~cm})$.

The variety having different plant height, cluster 1 and 3 (Table 3 ) could be effectively utilized in developing crosses/ selection of genotypes with better plant height because in case of jute it is the most important yield contributing character. Jatothu et al., (2018) conducted an experiment to examine the magnitude of genetic diversity and characters contributing to genetic diversity among 81 tossa jute genotypes. From the other traits studied like basal diameter it was recorded (Table 1) that white jute have more basal diameter $(1.51 \mathrm{~cm})$ than tossa jute $(1.36 \mathrm{~cm})$. This could mean that tall and lanky plants of C. olitorius (tossa jute) indicated more chance to lodge following the late showers during
August prior to its harvest. Selection for plants with more basal girth would be desirable in case of $C$. olitorius. However in the $C$. capsularis (white jute) highest basal diameter was noted in CIJ $26(1.73 \mathrm{~cm})$ followed by JRC $698(1.70 \mathrm{~cm})$ and in olitorius it was OIJ $18(1.54 \mathrm{~cm})$ followed by OIJ $22(1.48 \mathrm{~cm})$. In case of green wt per plant the mean was more in case of tossa jute $172.33 \mathrm{~g}$ but the range was more in white jute (101.87 gm - $246.75 \mathrm{gm})$, which indicated that there was enough opportunity for selection for this trait. The same trend was seen in case of fibre weight which is quite as expected. The mean fibre weight was $11.71 \mathrm{gm}$ in tossa jute and 8.85 gm in white jute whereas the range was more in $C$. capsularis $(13.01 \mathrm{gm}-4.89 \mathrm{gm})$. This also indicates one possibility that though there was considerable variability in both the species but responce to selection would be more in tossa jute and the fibre $\%$ was more in some C.olitorius lines like OIN -19, OIJ-06 and OIJ-07.

Table.1 Overall mean performances of different traits in two cultivated species

\begin{tabular}{|c|c|c|c|c|c|c|c|}
\hline & & \multicolumn{3}{|c|}{ C. capsularis } & \multicolumn{3}{c|}{ C. olitorius } \\
\hline $\begin{array}{c}\text { SL } \\
\text { NO }\end{array}$ & Traits & Average & Highest & Lowest & Average & Highest & Lowest \\
\hline $\mathbf{1}$ & Plant height (cm) & 255.60 & 317.16 & 174.70 & 294.66 & 327.33 & 245.33 \\
\hline $\mathbf{2}$ & $\begin{array}{c}\text { Basal Diameter } \\
(\mathrm{cm})\end{array}$ & 1.51 & 1.725 & 1.18 & 1.36 & 1.54 & 1.22 \\
\hline $\mathbf{3}$ & $\begin{array}{c}\text { Green Weight } \\
\text { (gm) }\end{array}$ & 169.44 & 246.75 & 101.87 & 172.33 & 222.40 & 113.26 \\
\hline $\mathbf{4}$ & Fibre weight (gm) & 8.85 & 13.01 & 4.89 & 11.71 & 15.45 & 7.79 \\
\hline $\mathbf{5}$ & Fibre \% & 5.03 & 6.03 & 3.85 & 6.82 & 8.28 & 5.57 \\
\hline
\end{tabular}

Table.2 Character association studies between fibre yield and other traits

\begin{tabular}{|c|c|c|c|c|c|}
\hline Species & Traits & $\begin{array}{c}\text { Plant } \\
\text { height }\end{array}$ & $\begin{array}{c}\text { Basal } \\
\text { diameter }\end{array}$ & $\begin{array}{c}\text { Green } \\
\text { weight }\end{array}$ & Fibre \% \\
\hline C. olitorius & Fibre yield & $0.768 * *$ & $0.873 * *$ & $0.881 * *$ & $0.546 * *$ \\
\hline C. capsularis & & $0.328 * *$ & $0.633 * *$ & $0.801 * *$ & $0.330 * *$ \\
\hline
\end{tabular}


Table.3 The group of 154 lines was divided into three clusters on the basis of genetic dissimilarity. The results are given below

\begin{tabular}{|c|c|c|}
\hline $\begin{array}{c}\text { SL } \\
\text { NO }\end{array}$ & Lines/ accessions & Group characteristics \\
\hline 1 & $\begin{array}{l}\text { CIN-01, CIN-07, CIN-08, CIN-12, CIN-16*, CIN-18, } \\
\text { CIN-19, CIN-21, CIN-22, CIN-23, CIN-44, CIN-46, CIN- } \\
\text { 52, CIN-54, CIN-56, CIN-66, CIN-74, CIJ-01, CIJ-05, } \\
\text { CIJ-09, CIJ-14, CIJ-23, CIJ-26, CIJ-31, JRC-517, JRC- } \\
\text { 698, OIN-02, OIN-07, OIN-10, OIN-13, OIN-16, OIN-21, } \\
\text { OIN-23, OIN-24 OIN-28, OIN-29, OIN-31, OIN-33, OIN- } \\
\text { 35, OIN-37, OIN-39, OIN-42, OIN-43, OIN-44, OIN-45, } \\
\text { OIN-46 OIN-47, OIN-51, OIN-56, OIN-57, OIN-58, OIN- } \\
\text { 66, OIN-70, OIN-75, OIN-78, OIN-79, OIN-80, OIN-81, } \\
\text { OIN-82, OIN-85, OIJ-02, OIJ-03, OIJ-04, OIJ-07, OIJ-08, } \\
\text { OIJ-09, OIJ-10, OIJ-11, OIJ-14, OIJ-15, OIJ-18, OIJ-20, } \\
\text { OIJ-22, , OIJ-23, OIJ-24, OIJ-27, OIJ-29, JRO 524, JRO } \\
\text { 204 [*CIN } 16 \text { is the central object] }\end{array}$ & $\begin{array}{c}\text { Total } 79 \text { lines. } \\
\text { Plant Height }(\mathrm{cm}) 293.38, \\
\text { Basal diameter }(\mathrm{cm}) 1.45, \\
\text { Green weight }(\mathrm{gm}) 189.46, \\
\text { Fibre weight }(\mathrm{gm}) 11.86, \\
\text { Fibre } \% 6.28 . \\
\text { Note: } 15.97 \text { gm of green wt is } \\
\text { responsible for } 1 \text { gm dry fibre } \\
\text { production. }\end{array}$ \\
\hline 2 & $\begin{array}{l}\text { CIN-03, CIN-04, CIN-05, CIN-14, CIN-24, CIN-25, CIN- } \\
\text { 27, CIN-28, CIN-29, CIN-30, CIN-31, CIN-32, CIN-33, } \\
\text { CIN-34, CIN-35, CIN-36, CIN-37, CIN-38, CIN-39, CIN- } \\
\text { 41, CIN-42, CIN-49, CIN-51, CIN-57, CIN-61, CIN-62, } \\
\text { CIN-63, CIN-70, CIN-71, CIN-72, CIN-73, CIN-79, CIN- } \\
\text { 83, CIJ-02, CIJ-03, CIJ-06, CIJ-08, CIJ-10, CIJ-11, CIJ- } \\
\text { 12, CIJ-13, CIJ-16, CIJ-19, CIJ-21, CIJ-22, CIJ-25, CIJ- } \\
\text { 27, CIJ-29*, CIJ-30, CIJ-32,OIN-04, OIN-11, OIN-53, } \\
\text { OIN-64, OIJ-21 [*CIJ-29 16 is the central object] }\end{array}$ & $\begin{array}{c}\text { Total } 55 \text { lines. } \\
\text { Plant height }(\mathrm{cm}) 241.72, \\
\text { Basal diameter }(\mathrm{cm}) 1.40, \\
\text { Green weight }(\mathrm{gm}) 153.43 \text {, } \\
\text { Fibre weight }(\mathrm{gm}) 8.04, \\
\text { Fibre } \% 5.25 . \\
\text { Note: } 19.08 \mathrm{~g} \text { of green wt is } \\
\text { responsible for } 1 \mathrm{~g} \text { fibre wt }\end{array}$ \\
\hline 3 & $\begin{array}{l}\text { CIJ }-04, \text { OIN-05, OIN-12, OIN-14, OIN-19, OIN-26, OIN- } \\
\mathbf{2 7 *} \text {, OIN-34, OIN-36, OIN-54, OIN-55, OIN-61, OIN-67, } \\
\text { OIJ-01, OIJ-05, OIJ-06, OIJ-16,OIJ-17, OIJ-19, OIJ-28 } \\
\text { [*OIN } 27 \text { is the central object] }\end{array}$ & $\begin{array}{l}\text { Total } 20 \text { lines. } \\
\text { Plant height }(\mathrm{cm}) 295.01, \\
\text { Basal diameter }(\mathrm{cm}) 1.32, \\
\text { Green weight }(\mathrm{gm}) 145.58, \\
\text { Fibre weight }(\mathrm{gm}) 10.25, \\
\text { Fibre } \% 7.06 . \\
\text { Note: } 14.20 \mathrm{~g} \text { of green } w t \text { is } \\
\text { responsible for } 1 \mathrm{~g} \text { fibre } \mathrm{wt}\end{array}$ \\
\hline
\end{tabular}


Table.4 Mean performances of different morpho-agronomic traits of $C$. capsularis genotypes

\begin{tabular}{|c|c|c|c|c|c|c|}
\hline SI No & Genotypes & $\begin{array}{c}\text { Plant } \\
\text { height }(\mathbf{c m})\end{array}$ & $\begin{array}{c}\text { Basal } \\
\text { diameter } \\
(\mathrm{cm})\end{array}$ & $\begin{array}{l}\text { Green wt } \\
\quad(\text { gm) }\end{array}$ & $\begin{array}{l}\text { Fibre wt } \\
\quad(\text { gm) }\end{array}$ & Fibre \% \\
\hline 1 & CIN-01 & 295.0 & 1.49 & 195.26 & 10.41 & 5.27 \\
\hline 2 & CIN-03 & 235.7 & 1.54 & 169.44 & 9.92 & 5.85 \\
\hline 3 & CIN-04 & 275.7 & 1.31 & 137.04 & 7.54 & 5.52 \\
\hline 4 & CIN-05 & 198.5 & 1.20 & 102.75 & 5.10 & 5.01 \\
\hline 5 & CIN-07 & 288.3 & 1.56 & 222.22 & 11.87 & 5.33 \\
\hline 6 & CIN-08 & 283.7 & 1.51 & 185.58 & 10.17 & 5.49 \\
\hline 7 & CIN-12 & 295.5 & 1.47 & 189.09 & 9.03 & 4.82 \\
\hline 8 & CIN-14 & 215.8 & 1.20 & 128.76 & 7.35 & 5.64 \\
\hline 9 & CIN-16 & 293.0 & 1.60 & 191.05 & 11.09 & 5.79 \\
\hline 10 & CIN-18 & 286.8 & 1.49 & 179.02 & 10.48 & 5.86 \\
\hline 11 & CIN-19 & 271.8 & 1.34 & 161.23 & 8.66 & 5.37 \\
\hline 12 & CIN-21 & 290.3 & 1.54 & 187.48 & 11 & 5.87 \\
\hline 13 & CIN-22 & 310.7 & 1.64 & 202.81 & 11.64 & 5.72 \\
\hline 14 & CIN-23 & 276.8 & 1.52 & 187.67 & 8.95 & 4.84 \\
\hline 15 & CIN-24 & 185.2 & 1.22 & 123.30 & 4.90 & 3.95 \\
\hline 16 & CIN-25 & 174.7 & 1.18 & 101.87 & 4.93 & 4.92 \\
\hline 17 & CIN-27 & 261.5 & 1.48 & 188.33 & 9.825 & 5.21 \\
\hline 18 & CIN-28 & 241.5 & 1.39 & 196.12 & 8.32 & 4.25 \\
\hline 19 & CIN-29 & 227.2 & 1.36 & 155.86 & 7.29 & 4.64 \\
\hline 20 & CIN-30 & 177.8 & 1.42 & 137.96 & 5.31 & 3.85 \\
\hline 21 & CIN-31 & 233.7 & 1.40 & 156.30 & 7.87 & 5.14 \\
\hline 22 & CIN-32 & 267.0 & 1.32 & 129.89 & 6.72 & 5.13 \\
\hline 23 & CIN-33 & 229.5 & 1.26 & 126.47 & 5.74 & 4.77 \\
\hline 24 & CIN-34 & 252.7 & 1.44 & 159.65 & 7.03 & 4.41 \\
\hline 25 & CIN-35 & 216.2 & 1.33 & 145.02 & 6.33 & 4.35 \\
\hline 26 & CIN-36 & 216.7 & 1.43 & 149.71 & 6.51 & 4.33 \\
\hline 27 & CIN-37 & 221.0 & 1.45 & 176.65 & 7.64 & 4.41 \\
\hline 28 & CIN-38 & 219.3 & 1.41 & 153.54 & 6.94 & 4.53 \\
\hline 29 & CIN-39 & 217.7 & 1.38 & 125.57 & 6.22 & 4.98 \\
\hline 30 & CIN-41 & 233.8 & 1.43 & 172.05 & 7.11 & 4.11 \\
\hline 31 & CIN-42 & 237.7 & 1.46 & 178.76 & 8.3 & 4.69 \\
\hline 32 & CIN-44 & 259.2 & 1.61 & 219.45 & 12.9 & 5.69 \\
\hline 33 & CIN-46 & 268.3 & 1.72 & 246.75 & 11.77 & 4.76 \\
\hline 34 & CIN-49 & 236.0 & 1.35 & 171.10 & 7.69 & 4.51 \\
\hline 35 & CIN-51 & 262.7 & 1.47 & 183.06 & 8.2 & 4.37 \\
\hline 36 & CIN-52 & 271.7 & 1.49 & 199.42 & 10.17 & 5.10 \\
\hline 37 & CIN-54 & 250.3 & 1.64 & 244.18 & 11.61 & 4.76 \\
\hline 38 & CIN-56 & 269.0 & 1.46 & 190.53 & 9.88 & 5.19 \\
\hline
\end{tabular}




\begin{tabular}{|c|c|c|c|c|c|c|}
\hline 39 & CIN-57 & 236.7 & 1.63 & 194.07 & 10.51 & 5.44 \\
\hline 40 & CIN-61 & 245.8 & 1.49 & 179.86 & 9.10 & 4.93 \\
\hline 41 & CIN-62 & 257.0 & 1.65 & 198.67 & 10.26 & 5.17 \\
\hline 42 & CIN-63 & 246.3 & 1.44 & 151.39 & 9.13 & 5.91 \\
\hline 43 & CIN-66 & 295.2 & 1.57 & 197.76 & 10.75 & 5.45 \\
\hline 44 & CIN-70 & 233.3 & 1.35 & 133.81 & 7.14 & 5.34 \\
\hline 45 & CIN-71 & 259.2 & 1.42 & 160.10 & 8.16 & 5.18 \\
\hline 46 & CIN-72 & 260.5 & 1.42 & 146.53 & 7.82 & 5.35 \\
\hline 47 & CIN-73 & 222.5 & 1.38 & 158.73 & 8.12 & 4.92 \\
\hline 48 & CIN-74 & 261.2 & 1.60 & 220.04 & 10.06 & 4.56 \\
\hline 49 & CIN-79 & 225.0 & 1.32 & 137.26 & 7.19 & 5.08 \\
\hline 50 & CIN-83 & 221.5 & 1.49 & 159.68 & 7.73 & 4.93 \\
\hline 51 & CIJ-01 & 317.2 & 1.55 & 193.85 & 10.82 & 5.58 \\
\hline 52 & CIJ-02 & 267.7 & 1.45 & 148.52 & 8.56 & 5.76 \\
\hline 53 & CIJ-03 & 250.7 & 1.56 & 156.98 & 8.63 & 5.50 \\
\hline 54 & CIJ-04 & 281.0 & 1.43 & 149.05 & 7.87 & 5.28 \\
\hline 55 & CIJ-05 & 286.3 & 1.58 & 190.96 & 10.82 & 5.69 \\
\hline 56 & CIJ-06 & 256.7 & 1.38 & 147.77 & 8.09 & 5.49 \\
\hline 57 & CIJ-08 & 259.5 & 1.38 & 151.82 & 8.57 & 5.60 \\
\hline 58 & CIJ-09 & 274.0 & 1.49 & 156.28 & 9.42 & 6.03 \\
\hline 59 & CIJ -10 & 250.0 & 1.41 & 164.83 & 9.72 & 5.83 \\
\hline 60 & CIJ -11 & 246.5 & 1.57 & 153.59 & 9.32 & 5.67 \\
\hline 61 & CIJ -12 & 260.8 & 1.41 & 149.36 & 7.83 & 5.15 \\
\hline 62 & CIJ -13 & 257.2 & 1.41 & 157.78 & 8.87 & 5.65 \\
\hline 63 & CIJ-14 & 269.0 & 1.62 & 171.74 & 9.89 & 5.77 \\
\hline 64 & CIJ-16 & 260.8 & 1.40 & 158.31 & 8.48 & 5.36 \\
\hline 65 & CIJ-19 & 257.3 & 1.41 & 132.87 & 8.01 & 5.99 \\
\hline 66 & CIJ-21 & 256.3 & 1.56 & 183.28 & 10.44 & 5.70 \\
\hline 67 & CIJ-22 & 256.7 & 1.57 & 197.53 & 10.84 & 5.52 \\
\hline 68 & CIJ-23 & 279.5 & 1.55 & 178.09 & 9.96 & 5.64 \\
\hline 69 & CIJ-25 & 244.0 & 1.31 & 109.63 & 5.89 & 5.36 \\
\hline 70 & CIJ-26 & 309.8 & 1.73 & 219.24 & 11.84 & 5.40 \\
\hline 71 & CIJ-27 & 258.5 & 1.42 & 144.63 & 8.25 & 5.74 \\
\hline 72 & CIJ-29 & 243.8 & 1.41 & 153.61 & 7.56 & 4.91 \\
\hline 73 & CIJ-30 & 259.5 & 1.59 & 194.89 & 10.75 & 5.52 \\
\hline 74 & CIJ-31 & 293.7 & 1.66 & 209.50 & 11.05 & 5.28 \\
\hline 75 & CIJ-32 & 259.7 & 1.44 & 155.19 & 7.8 & 5.08 \\
\hline 76 & JRC- $517^{+}$ & 304.7 & 1.56 & 186.95 & 10.98 & 5.86 \\
\hline \multirow[t]{4}{*}{77} & JRC- $698^{+}$ & 309.5 & 1.70 & 222.32 & 13.01 & 5.85 \\
\hline & Mean & 255.6 & 1.51 & 169.44 & 8.85 & 5.03 \\
\hline & Highest & 317.16 & 1.73 & 246.75 & 13.01 & 6.03 \\
\hline & Lowest & 174.7 & 1.18 & 101.87 & 4.89 & 3.85 \\
\hline
\end{tabular}


Table.5 Mean performances of different morpho-agronomic traits of C. olitorius genotypes

\begin{tabular}{|c|c|c|c|c|c|c|}
\hline SI No & Genotypes & $\begin{array}{c}\text { Plant } \\
\text { height }(\mathrm{cm})\end{array}$ & $\begin{array}{c}\text { Basal } \\
\text { diameter } \\
(\mathrm{cm})\end{array}$ & $\begin{array}{l}\text { Green wt } \\
\quad(\text { gm) }\end{array}$ & $\begin{array}{l}\text { Fibre wt } \\
\quad(\text { gm) }\end{array}$ & Fibre \% \\
\hline 1 & OIN-02 & 303.50 & 1.39 & 171.53 & 11.58 & 6.75 \\
\hline 2 & OIN-04 & 275.50 & 1.22 & 113.26 & 7.79 & 6.88 \\
\hline 3 & OIN-05 & 296.33 & 1.43 & 158.73 & 11.05 & 6.96 \\
\hline 4 & OIN-07 & 296.50 & 1.37 & 196.03 & 13.54 & 6.91 \\
\hline 5 & OIN-10 & 284.50 & 1.44 & 222.40 & 14.80 & 6.65 \\
\hline 6 & OIN-11 & 245.33 & 1.23 & 150.05 & 10.89 & 7.26 \\
\hline 7 & OIN-12 & 301.17 & 1.46 & 145.44 & 9.55 & 6.57 \\
\hline 8 & OIN-13 & 271.67 & 1.35 & 163.09 & 10.73 & 6.58 \\
\hline 9 & OIN-14 & 288.67 & 1.28 & 153.44 & 11.07 & 7.21 \\
\hline 10 & OIN-16 & 321.50 & 1.37 & 172.00 & 11.75 & 6.83 \\
\hline 11 & OIN-19 & 288.50 & 1.32 & 131.89 & 10.52 & 7.98 \\
\hline 12 & OIN-21 & 286.17 & 1.41 & 184.39 & 12.70 & 6.89 \\
\hline 13 & OIN-23 & 308.34 & 1.41 & 201.15 & 12.22 & 6.08 \\
\hline 14 & OIN-24 & 278.50 & 1.39 & 198.81 & 13.50 & 6.79 \\
\hline 15 & OIN-26 & 296.00 & 1.20 & 120.58 & 8.64 & 7.17 \\
\hline 16 & OIN-27 & 295.50 & 1.25 & 148.15 & 10.33 & 6.97 \\
\hline 17 & OIN-28 & 294.67 & 1.31 & 170.77 & 12.38 & 7.25 \\
\hline 18 & OIN-29 & 292.17 & 1.35 & 180.43 & 11.12 & 6.16 \\
\hline 19 & OIN-31 & 293.67 & 1.37 & 196.77 & 12.37 & 6.29 \\
\hline 20 & OIN-33 & 287.00 & 1.40 & 182.84 & 11.80 & 6.45 \\
\hline 21 & OIN-34 & 285.50 & 1.24 & 123.13 & 8.74 & 7.10 \\
\hline 22 & OIN-35 & 314.17 & 1.40 & 177.62 & 9.70 & 5.46 \\
\hline 23 & OIN-36 & 286.67 & 1.32 & 146.71 & 10.07 & 6.86 \\
\hline 24 & OIN-37 & 275.17 & 1.46 & 182.10 & 10.14 & 5.57 \\
\hline 25 & OIN-39 & 290.34 & 1.42 & 204.29 & 13.73 & 6.72 \\
\hline 26 & OIN-42 & 304.33 & 1.37 & 185.01 & 12.75 & 6.89 \\
\hline 27 & OIN-43 & 304.00 & 1.41 & 201.90 & 13.38 & 6.63 \\
\hline 28 & OIN-44 & 294.50 & 1.38 & 187.49 & 11.40 & 6.08 \\
\hline 29 & OIN-45 & 296.83 & 1.45 & 210.50 & 12.28 & 5.83 \\
\hline 30 & OIN-46 & 294.17 & 1.47 & 208.97 & 13.09 & 6.26 \\
\hline 31 & OIN-47 & 306.83 & 1.41 & 167.64 & 11.31 & 6.75 \\
\hline 32 & OIN-51 & 283.84 & 1.35 & 181.40 & 12.54 & 6.91 \\
\hline 33 & OIN-53 & 266.83 & 1.23 & 140.53 & 8.76 & 6.23 \\
\hline 34 & OIN-54 & 299.34 & 1.35 & 155.21 & 10.35 & 6.67 \\
\hline 35 & OIN-55 & 297.33 & 1.29 & 155.01 & 9.43 & 6.08 \\
\hline 36 & OIN-56 & 315.00 & 1.31 & 180.75 & 13.27 & 7.34 \\
\hline 37 & OIN-57 & 283.50 & 1.33 & 172.67 & 10.71 & 6.20 \\
\hline 38 & OIN-58 & 285.33 & 1.39 & 175.21 & 11.18 & 6.38 \\
\hline
\end{tabular}




\begin{tabular}{|c|c|c|c|c|c|c|}
\hline 39 & OIN-61 & 297.34 & 1.31 & 158.37 & 11.65 & 7.36 \\
\hline 40 & OIN-64 & 261.67 & 1.23 & 120.45 & 7.95 & 6.60 \\
\hline 41 & OIN-66 & 300.84 & 1.35 & 176.80 & 13.31 & 7.53 \\
\hline 42 & OIN-67 & 289.00 & 1.29 & 149.87 & 9.99 & 6.67 \\
\hline 43 & OIN-70 & 286.83 & 1.43 & 189.73 & 13.67 & 7.20 \\
\hline 44 & OIN-75 & 322.00 & 1.46 & 195.80 & 12.62 & 6.45 \\
\hline 45 & OIN-78 & 274.67 & 1.31 & 161.84 & 11.74 & 7.25 \\
\hline 46 & OIN-79 & 269.50 & 1.29 & 187.80 & 10.78 & 5.74 \\
\hline 47 & OIN-80 & 278.50 & 1.32 & 170.10 & 10.77 & 6.33 \\
\hline 48 & OIN-81 & 295.00 & 1.36 & 167.58 & 10.36 & 6.18 \\
\hline 49 & OIN-82 & 310.83 & 1.37 & 187.48 & 12.45 & 6.64 \\
\hline 50 & OIN-85 & 308.33 & 1.40 & 173.66 & 10.09 & 5.81 \\
\hline 51 & OIJ-01 & 297.50 & 1.38 & 154.60 & 11.28 & 7.30 \\
\hline 52 & OIJ-02 & 296.17 & 1.44 & 186.52 & 13.09 & 7.02 \\
\hline 53 & OIJ-03 & 293.83 & 1.41 & 205.45 & 13.74 & 6.69 \\
\hline 54 & OIJ-04 & 282.50 & 1.42 & 193.08 & 13.97 & 7.24 \\
\hline 55 & OIJ-05 & 290.84 & 1.39 & 135.88 & 10.56 & 7.77 \\
\hline 56 & OIJ-06 & 300.67 & 1.31 & 135.82 & 10.76 & 7.92 \\
\hline 57 & OIJ-07 & 279.00 & 1.44 & 184.29 & 15.26 & 8.28 \\
\hline 58 & OIJ-08 & 316.50 & 1.37 & 173.79 & 13.28 & 7.64 \\
\hline 59 & OIJ-09 & 281.00 & 1.37 & 177.90 & 10.30 & 5.79 \\
\hline 60 & OIJ-10 & 279.84 & 1.34 & 163.77 & 11.65 & 7.11 \\
\hline 61 & OIJ-11 & 311.84 & 1.44 & 182.81 & 12.54 & 6.86 \\
\hline 62 & OIJ-14 & 302.83 & 1.38 & 179.69 & 11.79 & 6.56 \\
\hline 63 & OIJ-15 & 280.33 & 1.23 & 157.33 & 11.03 & 7.01 \\
\hline 64 & OIJ-16 & 304.34 & 1.24 & 137.04 & 9.80 & 7.15 \\
\hline 65 & OIJ-17 & 297.00 & 1.33 & 149.89 & 10.81 & 7.21 \\
\hline 66 & OIJ-18 & 314.50 & 1.54 & 212.94 & 15.45 & 7.26 \\
\hline 67 & OIJ-19 & 301.17 & 1.27 & 163.09 & 12.16 & 7.46 \\
\hline 68 & OIJ-20 & 316.83 & 1.41 & 160.05 & 12.24 & 7.65 \\
\hline 69 & OIJ-21 & 254.00 & 1.37 & 164.54 & 11.34 & 6.89 \\
\hline 70 & OIJ-22 & 308.50 & 1.48 & 194.48 & 12.09 & 6.22 \\
\hline 71 & OIJ-23 & 290.17 & 1.52 & 204.35 & 13.25 & 6.48 \\
\hline 72 & OIJ-24 & 327.17 & 1.45 & 194.39 & 10.92 & 5.62 \\
\hline 73 & OIJ-27 & 317.67 & 1.39 & 175.38 & 12.84 & 7.32 \\
\hline 74 & OIJ-28 & 306.17 & 1.29 & 139.65 & 10.41 & 7.45 \\
\hline 75 & OIJ-29 & 327.33 & 1.43 & 200.53 & 14.64 & 7.30 \\
\hline 76 & JRO-524+ & 314.50 & 1.44 & 195.01 & 14.89 & 7.64 \\
\hline \multirow[t]{4}{*}{77} & $\mathrm{JRO}^{2} 204^{+}$ & 313.50 & 1.39 & 190.19 & 14.86 & 7.81 \\
\hline & Mean & 294.66 & 1.36 & 172.33 & 11.71 & 6.82 \\
\hline & Highest & 327.33 & 1.54 & 222.40 & 15.45 & 8.28 \\
\hline & Lowest & 245.33 & 1.22 & 113.26 & 7.79 & 5.57 \\
\hline
\end{tabular}


However the most interesting parameter being the fibre\% was more in C.olitorius (mean was $6.82 \%$ ) and OIJ-07 recorded the highest fibre\% of 8.28 , this line also recorded the highest fibre wt of $15.26 \mathrm{~g}$. In case of C.capsularis mean fibre $\%$ was 5.83. Fibre percentage was lower in white jute [Kumar et al., 2005].

When the correlation was studied (Table 2) about the dry fibre weight with plant height, basal diameter, green wt and fibre $\%$, it showed significant positive correlation with all other traits. This result is same as Ngomuo et al., (2017). But the green weight was found high positive correlation in both the species. The green weight can be taken as indirect index for selection in the advance materials. This was in corroboration to the earlier findings that the capsularis genotypess subjected to acute moisture stress at early stage under field condition at Jhargram, West Bengal, gave 54-89\% more plant height and accumulated $67 \%$ more biomass compared to olitorius ones, but the growth difference narrowed down at the later stage as oltorius picked up growth with time, Mahaptra et al., 2009.

It was reported earlier that plant height and basal diameter, have high correlation with fibre yield and they were also identified as criteria for selection [Mahaptra et al., 2009]. But in this case it was found that in both C.olitorius and $C$. capsularis green weight showed a positive and high correlation to the fibre weight and this should be used as the selection criteria while handling the advance generation.

Since the seed and fibre purpose crops are grown during different environmental conditions, the seed crop can be further evaluated for these findings which will even help to identify the better lines with their actual evaluation for the fibre yield.
Among the three clusters maximum number of genotypes were contained in cluster 1 with 79 lines followed by cluster 2 (55 lines) and cluster 3 (20 lines). Overall mean plant height was maximum in cluster 3 and minimum in cluster 2. Maximum and minimum overall green weight was found in cluster 1 (189.46 gm) and cluster 3 (145.58 gm). Cluster 1 showed highest (11.86 gm) dry fibre weight whereas cluster 2 showed lowest (8.04 gm) overall dry fibre weight. Fibre recovery from green weight was highest in cluster 3. So, genotypes can be selected from theses three clusters for further crossing programme.

\section{Acknowledgements}

The authors would like to thank All India Network Project on Jute and Allied Fibres (AINP on J\&AF), Bidhan Chandra Krishi Viswavidyalaya, Nadia, West Bengal, India for financial support. The headquarter of AINPJ\&AF situated at Central Research Institute for Jute and Allied Fibres, Barrackpore, West Bengal.

\section{References}

Banerjee, S., Das, M., Mir, R.R., Kundu, A., Topdar, N., Sarkar, D.,Sinha, M.K., Balyan, H.S. and Gupta P.K. 2012. Assessment of genetic diversity and population structure in a selected germplasm collection of 292 genotypes by microsatellite (SSR) markers. Molecular Plant Breeding. 3: 11-25.

Hossain, A.M. and Sasmal, B.G. 2006. Heterosis for seed yield andcomponent character in Tossa Jute (Corchorus olitoriusL.). Agricultural Science Digest. 26:111-112.

Jatothu, J. L., Kumar, A. A., Choudhary, S. B., Sharma, H. K., Maruthi, R. T., Kar, C. S. \& Mitra, J. (2018). Genetic diversity analysis in tossa jute (Corchorus olitorius L.) germplasm lines. Journal 
of Applied and Natural Science. 10(1): $1-3$.

Kar, C.S., Kundu, A., Sarkar, D., Sinha, M.K. and Mahaptra, B.S. 2009. Genetic diversity in jute (Corchorus spp) and its utilization: a review. Indian Journal of Agricultural Sciences. 79 (8):575586.

Kumar, D., Das, A., Mahato, P., Lakshman, S.S. and Mandi, S. 2005. Identification key for notified varieties and varieties of common knowledge of jute (Corchorus olitorius L. and C. capsularis L.). The Indian Journal of Genetics and Plant Breeding. 65: 236238.

Kumar, D., Das, A., Mahato, P., Lakshman, S.S. and Mandi, S. 2006. Morphological characterization of jute varieties (Corchorus olitorius $\mathrm{L}$. and $\mathrm{C}$. capsularis L.) and their application for DUS testing. The Indian Journal of Genetics and Plant Breeding. 66: 319323.

Mahaptra, B.S.,Mitra, S., Ramasubramaniam,
T. and Sinha, M.K. 2009.Research on jute (Corchorus olitorius and $C$. capsularis) and kenaf (Hibiscus cannabinus and $H$. sabdariffa): present status and future perspective. Indian Journal of Agricultural Sciences. 79 (12): 951-967.

Mukherjee, S. and Kumar, D. 2002. Screening of tossa jute (Corchorus olitorius L.) germplasm for male sterility. The Indian Journal of Genetics and Plant Breeding. 62: 361-362.

Nayak, B. K. and Baisakh, B. 2009. Genetic analysis for fiber yield and its components in tossa jute (Corchorus olitorius L.). Environment and Ecology.27: 86-88.

Ngomuo, M., Stoilova, T., Feyissa, T. \& Ndakidemi, P. A. (2017). Characterization of Morphological Diversity of Jute Mallow (Corchorus spp.). International Journal of Agronomy. https://doi.org/10.1155/201 $7 / 6460498$.

\section{How to cite this article:}

Anita Roy, Sourav Hazari and Sudip Bhattacharya. 2020. Analysis of Comparative Genetic Diversity in Two Cultivated Species of Jute. Int.J.Curr.Microbiol.App.Sci. 9(01): 221-230. doi: https://doi.org/10.20546/ijcmas.2020.901.025 